\title{
Evolución de la Peritonitis Apendicular
}

En Cirugía Infantil la patología derivada del apéndice enfermo sigue constituyendo la principal causa de abdomen agudo, $(27,30,32)$ y la peritonitis apendicular su complicación más frecuente.

Con el transcurso de los años se ha podido apreciar un aumento considerable en el número de niños que presentan cuadros apendiculares complicados. $(34,35)$

Es así como en nuestro servicio es cada vez menor la incidencia de intervenciones por apendicitis aguda. En cambio, en el Servicio de Urgencia, las operaciones practicadas en nifios con diagnóstico de peritonitis apendicular muestran un incremento progresivo.

Pareciera que, actualmente, se es más reticente en la indicación operatoria de un cuadro de dolor en la fosa iliaca derecha, y se espeta que aparezcan signos de irritación peritoneal o de compromiso del estado general para enviar al nin̄o a operarse.

Por otra parte, sabido es que la mortalidad por peritonitis apendicular es 20 a 30 veces más elevada que en una apendicitis aguda simple. $(12,16,26)$

Estos hechos nos han lievado a analizar los factores que condicionan la evolución favorable o desfavorable de los niños operados por peritonitis de origen apendicular en el Hospital Roberto del Río.

\footnotetext{
* Servicio de Cirugía Infantil. Hospital Roburto del Rio.
}

\author{
Dis. Galicia Montecinos Latorre**, Gustavo \\ Aldunate Nocl** y Ricardo Ayala Munizaga**.
}

\section{MATERIAL Y METODO}

Se analizan las fichas clínicas de 159 enfermos operados entre Ios años 1968-1975, en los cuales se comprobó una peritonitis de origen apendicular.

Para valorar la gravedad inicial de la peritonitis, tomamos como parámetros el estado general y la intensidad del daño peritoneal encontrado en la operación, para definir tres diferentes grados de peritonitis:

Peritonitis grado 1: estado genetal conservado: apéndice gangrenado o perforado; supuración localizada en fosa jlíaca derecha.

Peritonitis grado 2: moderado compromiso del estado general; apéndice perforado; supuración en FID y/o en Douglas.

Peritonitis grado 3: grave compromiso del estado general; supuración peritoneal generalizada; abscesos múltiples intraabdominales.

Para calificar la evolución de estos pacientes tomamos como índices el tiempo de hospitalización, la existencia de complicaciones $\mathrm{y}$ de reintervenciones, y la mortalidad. Definimos así tres diferentes grados de evolución:

a) Buena: hospitalización menor de 10 días, sin complicaciones postoperatorias;

b) Regular: hospitalización entre 10 y 20 días, con complicacjones postoperatorias leves;

c) Mala: hospitalizacion superior a 20 días, con complicaciones postoperatorias graves que pueden Hevar incluso a reintervenciones, o a muerte. 


\section{RESULTADOS}

En nuestros 159 pacientes vemos que hay un franco predominio de los varones sobre las mujeres (Tabla No 1), y que el grupo etario más afectado corresponde a escolares. En menores de 2 años el cuadro es, felizmente, poco frecuente. Esto coincide con publicaciones de otros autores. $(3,7,18,28)$

Considerando la gravedad inicial, encontramos que el mayor número de casos correspondía a peritonitis grado 2. Comparativamente, el grado 3 alcanzó una baja incidencia (Tabia № 2).

Tabla N: 1

\section{EVOLUCION DE LAS PERITONI'TIS} APENDICULARES

(Hospital Roberto del Río. 196\&1973)

Edad y sexo en 159 enfermos

\begin{tabular}{lccc}
\hline Grupo elario & Niños & Niñas & Total casos \\
\hline Menos de 2 años & 3 & 3 & 5 \\
2 a 4 años & 15 & 6 & 21 \\
5 a 7 años & 33 & 9 & 42 \\
8 a 10 años & 27 & 22 & 49 \\
11 a 13 años & 32 & 10 & 42 \\
\hline Total & 110 & 59 & 159 \\
\hline
\end{tabular}

Tabla N:2

GRAVEDAD INICIAL

\begin{tabular}{lcc}
\hline Peritonitis & $N^{\alpha}$ casos & Porcentaje \\
\hline Grado 1 & 52 & $33 \%$ \\
Grado 2 & 67 & $42 \%$ \\
Grado 3 & 40 & $25 \%$ \\
\hline Total & & $100 \%$ \\
\hline
\end{tabular}

Hecho el diagnóstico y decidida la hospitalización, se tomaron las medídas preoperatorias habituales hasta llegar al acto quirúrgico (Tabla № 3). En 138 casos la vía de abordaje fue una laparotomía de Mc. Burney, haciendo otro tipo de laparotomía cuando el diagnóstico preoperatorio era dudoso. Así, en lactantes se usó una laparotomía transversa en 5 casos.

En nuestra revisión encontramos 2 casos en que no fue posible efectuar la apendicectomía por estar el apéndice totalmente necrosado. Sólo se efectuó reparación del munón cecal.

Lograda la apendicectonía y tratado el munón, se hizo aseo de la cavidad peritoneal para luego dejar drenajes. Damos extraordinaria importancia al tipo de drenaje usado, pensando en que, si hay un foco supurado intraabdominal, debe obligadamente comunicarse en buena forma con el exterior, para no tener complicaciones derivadas de la retención de pus. En 95 pacientes de nuestra serie encontramos un drenaje adecuado.

\section{Tabla $N: 3$ \\ EVOLUCION DE LA PERITONITIS APENDICULAR}

(Hospital Roberto del Rio)

Tratamiento quirúrgico en 159 enfermos
Laparatomia empleada

Mc. Burney

Vertical

Transversal

Apendicectomia

Efectuada

No cfectuada

Dienajes

Adecuados

Inadecuados
157

2

138

16

5

10

3

,, 75

1,25

95

59

$62 \quad 38$ 
Llamamos "drenaje adecuado" (Tabla No 4) al tubo de goma que drena FID a través de la herida operatoria, junto a otro similar dirigido a cualquiera otra colocación purulenta, exteriorizado por contrabertura lo más próximo al foco supurado, y mantenidos permeables por tiempo suficiente; la permeabilidad del tubo es fundamental, ya que es sabido que a las 24 horas la fibrina comienza a ocluir los orificios de él. Para evitar esto, se moviliza precozmente, o se instila suero fisiológico o soluciones de antibióticos, de uso discutible. $(15,24)$

Tabla N? 4

\section{CALIFICACION DEL DRENAJE USADO}

\section{Adecuado}

-tubo de goma blando

-drena FID (herida operatoria)

-drena otro foco (contrabertura)

- permeable siempre

-mantenido por tiempo suficiente

Inadecuado

-penrose solo

- no drena FID

- no drena otros focos

- salida muy alejada del foco

-retiro precoz

Al encontrar pus en el peritoneo se tomó una muestra para examen bacteriologico en 33 casos (Tabla N N 5); 28 muestras desarrollaron colonias, y 5 se informaron como "cultivos negativos". El germen más frecuentemente aislado fue E. Coli en cultivo puro, seguido de Klebsiella y Pseudomona. Diversos autores señalan que E. Coli es el microorganismo detectable con mayor frecuencia en las peritonitis apendiculares, puro o en mezcla de gếrmenes. $(1,3,12)$ Pero no debemos olvidar la existencia de anaerobios de distinto tipo que no desarrollan colonias en medios de cultivos corrientes $y$ que, por lo tanto, no se tipificaron en nuestra serie. $(12,26)$
Tabla N: 5

INFORME BACTERIOLOGICO (33 CASOS)

\begin{tabular}{lrc}
\hline 1) Cultivos positivos & 28 (mezcla de gérmenes) \\
-Escherichia Coli & 22 & veces \\
-Klebsiella & 3 & $" \prime$ \\
-Pseudononas & 2 & $"$ \\
-Staph. Epidermidis & 2 & $"$ \\
-B. Pseudocarburn- & & \\
coso & 1 & $"$ \\
- Staph. Dorado & 1 & \\
-Aerobacter Aeró- & & \\
genes & 1 & $"$ \\
2) Cultivos negativos & 5 & \\
3) Total de cultivos & 33
\end{tabular}

Ya sea como indicación preoperatoria, o en el postoperatorio inmediato, todos los pacientes recibieron algún tipo de antibióticos por vía de general (Tabla No 6).

Según las disponibilidades del servicio, la combinación penicilina-quemicetina se usó en 124 casos, seguida de ampicilina.

Por estimar que la respuesta clínica al antibiótico no fue satisfactoria, se cambió de medicamento en 66 casos. El nuevo antibiótico fue, preferentemente, ampicilina (Tabla N:7).

Tabla N: 6

\begin{tabular}{lr} 
ANTIBIOTICOS USADOS EN & 159 ENFERMOS \\
\hline Tipo de anfibiótico & No de enfermos \\
Penicilina-quemicetina & 124 casos \\
Ampicilina & $21 " \prime$ \\
Kanamicina & $6 \% "$ \\
Gentamicina & $2 "$ \\
Se cambió antibiótico en & 66 casos \\
\hline
\end{tabular}

Tabla No 7

ANTIBIOTICOS USADOS EN SEGUNDA INDICACION

\begin{tabular}{|c|c|c|}
\hline Tipo de antibibtico & No $d e$ & enfermos \\
\hline Ampicilina & 52 & casos \\
\hline Kanamicina & 16 & $"$ \\
\hline Gentamicina & 8 & $"$ \\
\hline Wintomylon & 3 & $"$ \\
\hline Neomicina & 4 & " \\
\hline Dicloxacilina & 2 & " \\
\hline Septrin & 2 & $"$ \\
\hline
\end{tabular}


Hemos querido comparar nuestros resultados usando antibióticos en todos los casos estudiados, con series de autores extranjeros (Tabla N N 8), específicamente de Shandling, $26 \mathrm{y}$ de Stanley-Brown, 28. Encontramos que, con un $100 \%$ de uso de antibióticos, tenemos la misma cantidad de abscesos intrabdominales, pero un mayor porcentaje de infec- ción de la herida operatoria. Nos preguntamos entonces: ¿Son verdaderamente necesarios los antibióticos cuando el foco séptico abdominal se ha eliminado? ¿No sería mós conveniente esperar la evolución postoperatoria para indicarlos cuando el cuadro clínico nos diga que realmente se necesitan?

Tabla $N^{0} 8$

COMPARACION DE CASUISTICAS (complicaciones y uso de antibiot.)

\begin{tabular}{lcccc}
\hline Autor & No casos & $\begin{array}{c}\text { \% infeccion } \\
\text { herida op. }\end{array}$ & $\begin{array}{c}\% \text { abscesos } \\
\text { intrabdom. }\end{array}$ & $\begin{array}{c}\% \text { usa de } \\
\text { antibibt. }\end{array}$ \\
\hline $\begin{array}{l}\text { Longino } \\
1958\end{array}$ & 611 & 7,3 & 9,4 & 100 \\
$\begin{array}{c}\text { Boles } \\
1959\end{array}$ & 252 & 9,9 & 15,9 & 100 \\
$\begin{array}{l}\text { Fock } \\
1969\end{array}$ & 545 & 7,5 & 16,0 & 98 \\
$\begin{array}{l}\text { St. Brown } \\
1971\end{array}$ & 100 & 24,0 & 17,0 & 95 \\
$\begin{array}{l}\text { Shandling } \\
1974\end{array}$ & 550 & 18,7 & 13,6 & 35 \\
Serie actual & 159 & 29,0 & 12,0 & 100 \\
1975 & & & & \\
\hline
\end{tabular}

Nos interesó saber también qué influencia tenía el tiempo de evolución pre-operatoria sobre la gravedad inicial del cuadro (Tabla No 9). Encontranos que las peritonitis más graves tenían mayor cantidad de enfermos con 3 o más días de evolución con respecto al momento del diagnóstico adecuado. En 22 enfermos este retardo en el diagnóstico se debió a una interpretación equivocada del cuadro clínico (Tabla $\mathrm{N}$ 10): más o menos 1 de cada 8 enfermos había recibido tratamiento médico inadecuado en base a antibióticos, generalmente de amplio espectro, por tiempo variable. Los errores diagnósticos nás frecuentes fueron: estado infeccioso $\mathrm{zt}$ foidea? y gastroenteritis.

Tabla $N^{a} \mathbf{9}$

TIEMPO DE EVOLUCION Y GRAVEDAD INICIAL

\begin{tabular}{lccc}
\multicolumn{2}{c}{ Tiempo de } & \multicolumn{3}{c}{ Peritonitis } \\
evolución preoper. & Grado 1 & Grado 2 & Grado 131 \\
\hline Menos de 24 horas & 14 & 8 & 6 \\
1 a 2 dias & 27 & 23 & 7 \\
3 dias o mass & 12 & 29 & 26 \\
\hline
\end{tabular}


COMPLICACIONES ENCONTRADAS

EN 93 ENFERMOS

ERRORES DIAGNOSTICOS QUE RETARDARON EL INGRESO

Tipo de diagnóstico

Núntero de casos

rslado infeccioso itifoidea?

Castroenteritis-diarrea

Fstado gripal

Cólico renal

Pútpura Schonlsin-Hettoch

Infección urinaria

Pancreatitis

Sepsis

Total

$\begin{array}{r}6 \\ 6 \\ 3 \\ 2 \\ 1 \\ 2 \\ 1 \\ 1 \\ \hline 22\end{array}$

La evolución de la peritonitis apendicular está marcada por la existencia de complicaciones que, invariablemente, ocurren en estos enfermos en porcentaje considerable. En nuestra serie encontramos que 93 pacientes $(58 \%)$ presentaton algún tipo de complicación postoperatoria, siendo los niños de menor edad los que más se complicaron; por io tanto, es lógico suponer que su recuperación es más lenta.

Entre las complicaciones encontradas, la más frecuente fue d absceso residual (Tabla No 11), con 65 casos. De éstos, 46 casos corresponden a la herida operatoria, y 15 a abscesos intrabdom in a I e s. Probablemente llame la atención la gran frecuencia de infección de la herida operatoria, pero fuimos extraordinariamente estrictos en la califica. ción de esta complicación.

El absceso intrabdominal causó la mayoría de los cuadros obstructivos, o fue factor coadyuvante en al mantención de ileos pa. ralíticos prolongados. Algunos de estos últimos presentaron diarreas graves en el período de recuperación de la motilidad intestinal, llegando un caso a la deshidratación franca.

\begin{tabular}{lcc}
\hline Tipo de complicación & No de veces & $\%$ \\
\hline & 65 & 40 \\
Absceso residual & 46 & 29 \\
- cn herida operatoria & 19 & 12 \\
-intrabdominal & 11 & 6,2 \\
lieo paralítico prolongado & 18 & 10,3 \\
Jleo mecánico & 8 & 5,1 \\
Sepsis grave & 9 & 5,3 \\
Complicaciones pulmonares & 5 & 3,1 \\
Evisceración-cventración & 25 & 15,6 \\
Otras complicaciones & &
\end{tabular}

Hemos confirmado una vez más que en cualquiera de los tres diferentes grados de evolución señalados al comienzo, influye lógicamente la gravedad inicial del compromiso peritoneal. Los niñ́os que se operan con peritonitis más avanzada tienen más mala evolución que aquellos con peritonitis en Grado 1, a pesar de recibir igual manejo quirúrgico.

Tabla N: 12

GRAVEDAD INICIAL Y EVOLUCION

\begin{tabular}{lccc}
\hline & Buena & Regular & Mala \\
\hline & & & \\
Pcritonitis grado 1 & $49 \%$ & $40 \%$ & $11 \%$ \\
Peritonitis grado 2 & $42 \%$ & $38 \%$ & $20 \%$ \\
Peritonitis grado 3 & $30 \%$ & $40 \%$ & $30 \%$ \\
\hline
\end{tabular}

Resulta también interesante señalar que las peritonitis más graves tienden a hacer complicaciones más frecuentes, especialmente en lactantes. Es justamente en este grupo etario en donde se registraron los dos casos fatales.

\section{CONCLUSIONES}

Del análisis de nuestros resultados podemos concluir que:

1? Existe una alta incidencia de peritonitis apendicular en nuestro Servicio; 
2 . Por razones poco claras, afecta más a hombres que a mujeres;

3. Evolucionan peor: a) los niños de menor edad; b) las mujeres que los hombres, por factores que desconocenos; c) los pacientes con tiempo de evolución preoperatorio más prolongado; d) los enfermos con diagnóstico hecho tardíamente; e) los niños con tratamiento quirúrgico inadecuaco, especialmente cuando el drenaje de los focos supurados peritoneales ha sido insuficiente o inadecuado.

4\% Los antibióticos muchas veces retardan el diagnóstico (por uso indiscriminado pensando en cuadros médicos); no impiden las complicaciones, $y$ por lo tanto no parecen disminuir la morbilidad.

\section{RESUMEN}

Se analiza la evolución de 159 niños operados en los que se comprobó una peritonitis apendiculat.

Se estudia la correlación entre la evolución favorable o desfavorable con factores como: edad, sexo, días de evolución preoperatoria, complicaciones, uso de drenajes y de antibióticos, etc.

\section{SUMMARY}

The evolution of 159 operated children, in whom and appendiceal peritonitis was found, is analized.

The correlation between the favorable and unfavorable evolution with factors like: sex, age, days of preoperative evolution, complication, use of antibiotics, drainage, etc., are studied.

\section{REFERENCIAS}

1. Atwhein, E.E. et at. Ped, Clin. of N.A. 6: 77.80, 1959.

2. Barabas, A.P. Br. J. Clin. Pract. 24: 489-491, 1970.

3. Blair Gl. et al. J. Ped. Surg. 4: 445, 1969.
4. Broadbent, Nr. ef at. Austr. N.Z. ]. Surg. 40: 362, 1971.

5. Bryant, T.L. Amer. Surg. 36: 523, 1970.

6. Bartlett. R.H. et al. Year Book of General Surgery, 1970. Resumen.

7. Cumacho, E. y cols. Rev. Chil. Ped. 32: 344, 1961.

8. Daudet, M. et at. Ant. Chir. Inf. 6: 155, 1965.

9. Dicescu, T. et al. Ped. 21: 251, 1972.

10. Gaillard, L, et al. Pediatrie 26: 438, 1971 .

11. Gsatrin, U. et al. Actha Chir. Scand. 136: 153, 1970.

12. Gunnar, F. et al. Year Book of General Surgery, 1970.

13. Gross, $R$. "Surgery in infancy and childhood". pp. 253 W.B. Saunders Co., 1953.

14. Holgersen, L.D. et al. J. Ped. Surg. 5: 379, 1970.

15. Jerveth, T.C. et al. J. Ped. Sur. 6: 121, 1971.

16. Kiessewetter, W.B. Amer. Fam. Physician, 5: 105, 1972 .

17. Liebman, W.M. et al. Amer. J. Dis. Child. 120: 77, 1970.

18. Longino, L.A. et al. Ped, 22: 238, 1958.

19. Parsons, J.L. et al. Sur. 67: 841, 1967.

20. Pellerin, D. et al. Maroc. Med. 50: 575, 1970.

21. Pereyra, H. y cols. Acta Gast. Lat. Am. 4; 27, 1972.

22. Philips, S.J. et al. N.Y. State J. Med. 71: 985-86. 1971.

23. Potts, W.J. Surgeon and Child. Phyladelphia, W.B. Saunders Co. 1959, pp. 185-194.

24. Raffensperger, J.G. et al, Chicago Med. Sch. Quart. 28: 179-83, 1969.

25. Lowe, M.J. Ped. 38: 1057-59, 1966.

26. Shanding, B. et al. J. Ped. Surg. 9: 79-83, 1974.

27. Singer, B. et al. S. Afr. Med. J. 46: 987-93. 1972.

28. Stantey-Brown, E.G. Am. J. Dis, Child. 108: 134-38, 1964.

29. Sione, H.H. et al. Surg. 69: 673-79, 1971.

30. Tabrisky, J. et al. Amer. J. Dis Child. 111: 557-59, 1966.

31. Thompson, W.M. et al. J. Ped. Sur. 6: 458-61, 1971.

32. Thompson, W.M. et al. Ped. 38: 1057-59, 1966.

33. Vera, M. y cols. Arch. Soc. Cir. Chile, 13: 429 . 37, 1961.

34. Vera, M. y cols. Rev. Chil. Ped. 36: 230-35, 1965.

35. Vers, M. y cols, Rev. Chil. Ped. 34: 189-95, 1963.

36. Vera, $M$, y cols. Rev. Chil. Ped. 42: 121-24, 1971. 